\title{
Homotopy perturbation based linearization of nonlinear heat transfer dynamic
}

\author{
S.H. Hosseinnia • A. Ranjbar · D.D. Ganji • \\ H. Soltani · J. Ghasemi
}

Received: 14 May 2008 / Revised: 24 June 2008 / Published online: 25 July 2008

(C) The Author(s) 2008. This article is published with open access at Springerlink.com

\begin{abstract}
In this paper a new linearization procedure based on Homotopy Perturbation Method (HPM) will be presented. The procedure begins with solving nonlinear differential equation by HPM. This will be done by evaluation of the time response of a nonlinear dynamic. An equivalent Laplace transform of the time response will be obtained. In the preceding, the effect of an external excitation i.e. input, will be removed from the model to find an approximate linear model for the nonlinear dynamic. The effectiveness of the procedure is verified using a heat transfer nonlinear equation. Ultimately, both HPM based linear model and that of nonlinear have been controlled via a closed loop PID controller. The simulation result shows the significance of the proposed technique.
\end{abstract}

Keywords Dynamic $\cdot$ Linearization $\cdot$ Heat transfer $\cdot$ Homotopy perturbation

Mathematics Subject Classification (2000) 35N05 · 35B20

\section{Introduction}

System is usually represented by a (set of) differential equation. To model the whole process, a complex and almost nonlinear differential equation is inevitable. The nonlinear dynamic has to be solved to find out the actual behavior in different circumstances. An analytical solution or a direct answer is usually difficult to find. Therefore an approximation approaches such as (HPM, HAM and VIM) might be of alternatives [1-17].

S.H. Hosseinnia · A. Ranjbar · D.D. Ganji $(\bowtie) \cdot$ H. Soltani · J. Ghasemi Faculty of Electrical and Mechanical Engineering, Noushirvani University of Technology, P.O. Box 47135-484, Babol, Iran e-mail: ddg_davood@yahoo.com 
In the last two decades with the rapid development of researches toward analyzing nonlinear dynamics, there has been an ever-increasing interest of scientists and engineers in analytical techniques to solve nonlinear problems. The widely applied techniques (i.e. perturbation method) are of great interest to be used in control systems $[18,19]$. To eliminate the limitation of "small parameter", which is assumed in the perturbation method, a new technique based on the homotopy terminology $[5,6]$ has been proposed. Accordingly, a nonlinear problem is transformed into an infinite number of simple problems without using the perturbation technique. Effectively, letting the small parameter float and converge to the unity, the problem will be converted into a special perturbation problem with a small embedding parameter. Therefore the method receives the name, Homotopy Perturbation Method (HPM). The effectiveness of the new technique is presented in $[12,20]$. This approach can take full advantage of the traditional perturbation technique and Homotopy analysis method. The new scheme has been applied to linear and nonlinear ordinary and partial differential equations.

In this paper a step input is applied to a nonlinear heat transfer dynamic. The time response will be achieved using Homotopy Perturbation Method (HPM). The provided linear solution is transformed to a transfer function. Therefore a linear model is yielded based on HPM. The accuracy of the model will be undergoing to a verification procedure. The significance of the model is compared with the actual response of the nonlinear heat dynamic at the same circumstances. In the following a similar PID controller is applied to both dynamic which resulted with no more error.

\section{Basic idea of Homotopy Perturbation Method}

Let us introduce HPM via following nonlinear differential equation:

$$
A(x)-f(u)=0, \quad u \in \mathfrak{R}^{m} .
$$

Subjected to boundary conditions:

$$
B(x, \partial x / \partial t)=0, \quad x \in \Re^{n},
$$

where $A(x)$ is a general differential operator, $B(.,$.$) is a boundary operator, x$ is known as analytic and $n$ dimensional function (here, state) and $u$ is an $m$ dimensional input (independent variable). The differential part $A(x)$ can generally be divided into two linear $L(x)$, and nonlinear $N(x)$, parts. Equation (1) can therefore be rewritten as:

$$
L(x)+N(x)-f(u)=0 .
$$

A homotopy statement $H(v, p)$ using an auxiliary variable $v(u, p)$ with $p \in[0,1]$ will be defined as:

$$
H(v, p)=(1-P)\left[L(v)-L\left(x_{0}\right)\right]+p[A(v)-f(u)]=0, \quad p \in[0,1]
$$


$p$ is called the homotopy parameter. As $p$ equals $0.0,(4)$ is being completely linear whilst $p$ equals to 1.0 the linear part in (4) completely vanishes and (4) will become the same as (1). With a simple manipulation, (4) is reduced to the following equation:

$$
H(v, p)=L(v)-L\left(x_{0}\right)+p L\left(x_{0}\right)+p[N(v)-f(u)]=0, \quad p \in[0,1] .
$$

The initial guess $x_{0}$ needs to be a good initial approximation for the solution of (1). However, in the system area it is a property of the system and can be meaningfully found. An intelligent way to estimate $x_{0}$ is to assume it as the steady state value. However, it can be computed in advance if the whole system is stable. The solution of (4) may be expressed in terms of an infinite series as:

$$
v=p^{0} v_{0}+p^{1} v_{1}+p^{2} v_{2}+\cdots
$$

Replacing (6) in (5) and rearranging the resultant in terms of ascending powers of $p$, an infinite number of differential equations in terms of $v$, will be achieved. An extra attention should be taken into account to avoid the secular terms to produce boundedness [13]. This set of almost simpler differential equation is then solved using such proper initial conditions. Finally, an approximate solution of (1) can be written as:

$$
x=\lim _{p \rightarrow 1} v=v_{0}+v_{1}+v_{2}+\cdots .
$$

The convergence of HPM is discussed in [7, 10, 14]. Due to practical restrictions, a shorter term of $v_{i}, i=0,1,2, \ldots$ is of interest. This affects the accuracy of the solution, which in return produces some error. The result in (7) will be treated as a core to find a linear model for the nonlinear model.

\section{HPM based linearized model for nonlinear heat transfer equation}

In this section a HPM based linearization procedure will be given. The nonlinear heat transfer equation will be solved in time domain using HPM. Thereafter Laplace transform and some other technique will be used. The procedure will be expressed here.

\subsection{Cooling of a lumped system with variable specific heat}

The heat temperature of a ball with the specification in Table 1, at an initial temperature of $T_{I}$ is of interest. Due to low temperature the effect of radiation is not considered. The thermal dynamic is introduced here as [15-17]:

$$
\rho V c \dot{T}+h A\left(T-T_{a}\right)=0, \quad T(0)=T_{I}=1200 \mathrm{~K},
$$

where $T_{a}$ is the ambient temperature and $h$ is the convection constant. At this dynamic the specific heat $c$ is nonlinear. The variation can also be interpreted as:

$$
c=c_{a}\left[1+\beta\left(T-T_{a}\right)\right]
$$


Equation (9) is substituted in (8) and accordingly simplified. In terms of defined constant parameters, it can be written in (10) as:

$$
\dot{T}+k_{1} T+k_{2} T \dot{T}-k_{1} T_{a}=0, \quad T(0)=T_{I}=1200 \mathrm{~K},
$$

where constants parameters are evaluated at the ambient temperature as:

$$
\begin{aligned}
& k_{1}=\frac{h A}{\rho V c_{a}-\rho V c_{a} \beta T_{a}}=0.0222, \\
& k_{2}=\frac{\rho V c_{a} \beta}{\rho V c_{a}-\rho V c_{a} \beta T_{a}}=0.0013 .
\end{aligned}
$$

As it can be seen the dynamic is nonlinear. However the dynamic is excited by two terms of; an external forced temperature (i.e. external input, $T_{I}$ ) and initial condition. Therefore their effects will be spotted independently. Of course, it is of known that the superposition principle is not valid for nonlinear system. Since the objective is to find a best fit linear model of the nonlinear dynamic in (10), the separation is assumed valid. Consequently the dynamic response is evaluated, considering two different circumstances of:

I. Zero input response (under excitation of the initial condition)

II. Zero state response (under excitation of the external input).

Ultimately, the complete approximated response will be super positioned.

I. Zero input response: Equation (10) is again considered in presence of an initial condition.

$$
\underbrace{\dot{T}_{z i}+k_{1} T_{z i}}_{\text {Linear }}+\underbrace{k_{2} T_{z i} \dot{T}_{z i}}_{\text {Nonlinear }}=0, \quad T(0)=T_{I}=1200 \mathrm{~K},
$$

where $T_{z i}$ stands for the zero input response of the heat transfer equation. To gain the effectiveness of HPM, the linear and nonlinear parts are underlined respectively. Accordingly the Homotopy statement will be established as:

$$
H(p, v)=\dot{v}+k_{1} v-L\left(T_{0}\right)+p L\left(T_{0}\right)+p\left[k_{2} v \dot{v}\right]=0 .
$$

An initial approximation of $T_{0}=0$ is chosen such that the equation satisfies the steady state requirement. Considering $v=p^{0} v_{0}+p^{1} v_{1}+p^{2} v_{2}+\cdots$ incorporating the perturbation value in (12) yields as:

$$
\begin{aligned}
H(p, v)= & \left(p^{0} v_{0}+p^{1} v_{1}+p^{2} v_{2}+\cdots\right)+k_{1}\left(p^{0} v_{0}+p^{1} v_{1}+p^{2} v_{2}+\cdots\right) \\
& +p\left[k_{2}\left(p^{0} v_{0}+p^{1} v_{1}+p^{2} v_{2}+\cdots\right)\left(p^{0} v_{0}+p^{1} v_{1}+p^{2} v_{2}+\cdots\right)\right]=0 .
\end{aligned}
$$

Rearranging the equation in terms of ascending power of $p$ achieves the following equations:

$$
p^{0}: \quad \dot{v}_{0}+k_{1} v_{0}=0, \quad v_{0}(0)=1200,
$$




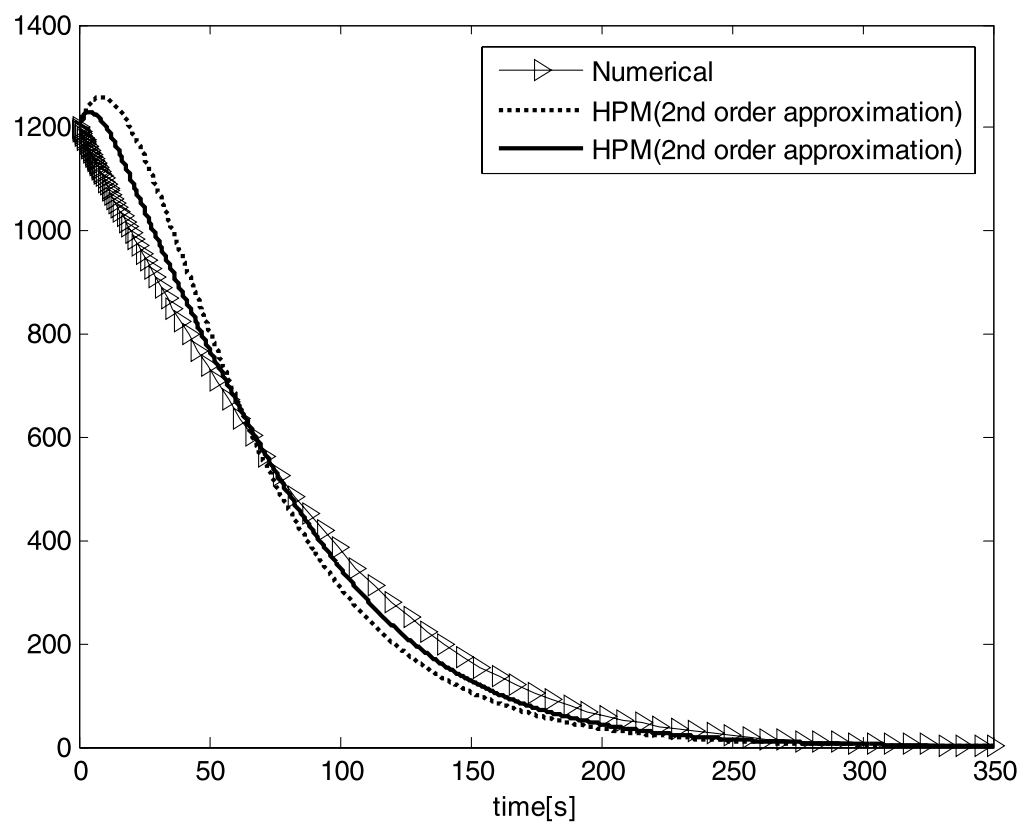

Fig. 1 HPM based Zero input equivalent considering 2nd and 3rd order $v$, with respect to the numerical one

$$
\begin{array}{ll}
p^{1}: & \dot{v}_{1}+k_{1} v_{1}+k_{2} v_{0} \dot{v}_{0}=0, \quad v_{1}(0)=0, \\
p^{2}: & \dot{v}_{2}+k_{1} v_{2}+k_{2}\left(v_{0} \dot{v}_{1}+v_{1} \dot{v}_{0}\right)=0, \quad v_{2}(0)=0 .
\end{array}
$$

These ordinary equations are then solved. To verify the effect of the series truncation in (7), a 2nd and 3rd order approximation for $v$ are chosen as:

$$
\begin{aligned}
& T_{2 n d}=\lim _{p \rightarrow 1}\left(v_{0}+p v_{1}\right), \\
& T_{3 r d}=\lim _{p \rightarrow 1}\left(v_{0}+p v_{1}+p^{2} v_{2}\right) .
\end{aligned}
$$

Therefore they are obtained as:

$$
\begin{aligned}
T_{2 n d}= & 3072 e^{-\frac{111}{5000} t}-1872 e^{-\frac{111}{2500} t} \\
T_{3 r d}= & 3072 e^{-\frac{111}{5000} t}-1872 e^{-\frac{111}{2500} t} \\
& +\left(\frac{-1898208}{625} e^{-\frac{333}{5000} t}+\frac{4270968}{625} e^{-\frac{111}{5000} t}\right. \\
& \left.-\frac{2847312}{625} e^{-\frac{111}{5000} t}+\frac{474552}{625}\right) e^{-\frac{111}{5000} t} .
\end{aligned}
$$

The appropriate responses are plotted together with the numerical in Fig. 1. 
During the transient time the approximation error is seen small but approaching to zero in the steady state. Furthermore the 3rd order approximation provides a better result. However both results are satisfactory and convincing.

II. Zero state equivalent: The second option is to find the effect of an independent input to the nonlinear system. At this stage the states are initially at rest. A step input is imposed to nonlinear differential equation. Equation (10) is again written assuming the relaxation under excitation of the input.

$$
\underbrace{\dot{T}_{i}+k_{1} T_{i}}_{\text {Linear }}+\underbrace{k_{2} T_{i} \dot{T}_{i}}_{\text {Nonlinear }}-\underbrace{k_{1} T_{a}}_{\text {input }}=0, \quad T(0)=0,
$$

where $T_{i}$ stands for the zero state equivalent response. For ease of reference, the linear nonlinear and input are respectively spotted in the equation. Accordingly the Homotopy statement is established as:

$$
H(p, v)=\dot{v}+k_{1} v-L\left(T_{0}\right)+p L\left(T_{0}\right)+p\left[k_{2} v \dot{v}-k_{1} T_{a}\right]=0 .
$$

The optional input is chosen as a step function of $T_{a}=300 \mathrm{~K}$. The steady state is also chosen as the initial approximation by $T_{0}=300 \mathrm{~K}$. The same procedure yields the following equation:

$$
\begin{aligned}
H(p, v)= & \left(p^{0} v_{0}+p^{1} v_{1}+p^{2} v_{2}+\cdots\right)+k_{1}\left(p^{0} v_{0}+p^{1} v_{1}+p^{2} v_{2}+\cdots\right) \\
& -k_{1} T_{0}+p k_{1} T_{0}+p\left[k_{2}\left(p^{0} v_{0}+p^{1} v_{1}+p^{2} v_{2}+\cdots\right)\right. \\
& \left.\times\left(p^{0} v_{0}+p^{1} v_{1}+p^{2} v_{2}+\cdots\right)-k_{1} T_{a}\right]=0 .
\end{aligned}
$$

The equation will be rearranged to the following equations:

$$
\begin{array}{lll}
p^{0}: & \dot{v}_{0}+k_{1} v_{0}-300 k_{1}=0, & v_{0}(0)=0, \\
p^{1}: & \dot{v}_{1}+k_{1} v_{1}+k_{2} v_{0} \dot{v}_{0}=0, & v_{1}(0)=0 .
\end{array}
$$

A 2nd order approximation of $v$ is chosen. Consequently it approaches to the following equation when $p$ approaches to unity.

$$
T_{i}=300-300 e^{-\frac{2}{125} t}+\left(\frac{7}{16} e^{\frac{2}{125} t}-\frac{3}{250} t+\frac{1865}{16}-117 e^{-\frac{2}{125} t}\right) e^{-\frac{2}{125} t} .
$$

The result is plotted in Fig. 2. The response with respect to the actual one confirms the quality and significance of 2nd order HPM based solution.

Since HPM provides a linear solution for the nonlinear dynamic, the provided responses from two different cases of I and II are summed up linearly. This constructs the complete HPM based linearized solution for the nonlinear system.

$$
\begin{aligned}
T_{2 n d}= & T_{i}+T_{z i 2 n d} \\
= & 300-300 e^{-\frac{2}{125} t}+\left(\frac{7}{16} e^{\frac{2}{125} t}-\frac{3}{250} t+\frac{1865}{16}-117 e^{-\frac{2}{125} t}\right) e^{-\frac{2}{125} t} \\
& +3072 e^{-\frac{111}{5000} t}-1872 e^{-\frac{111}{2500} t},
\end{aligned}
$$




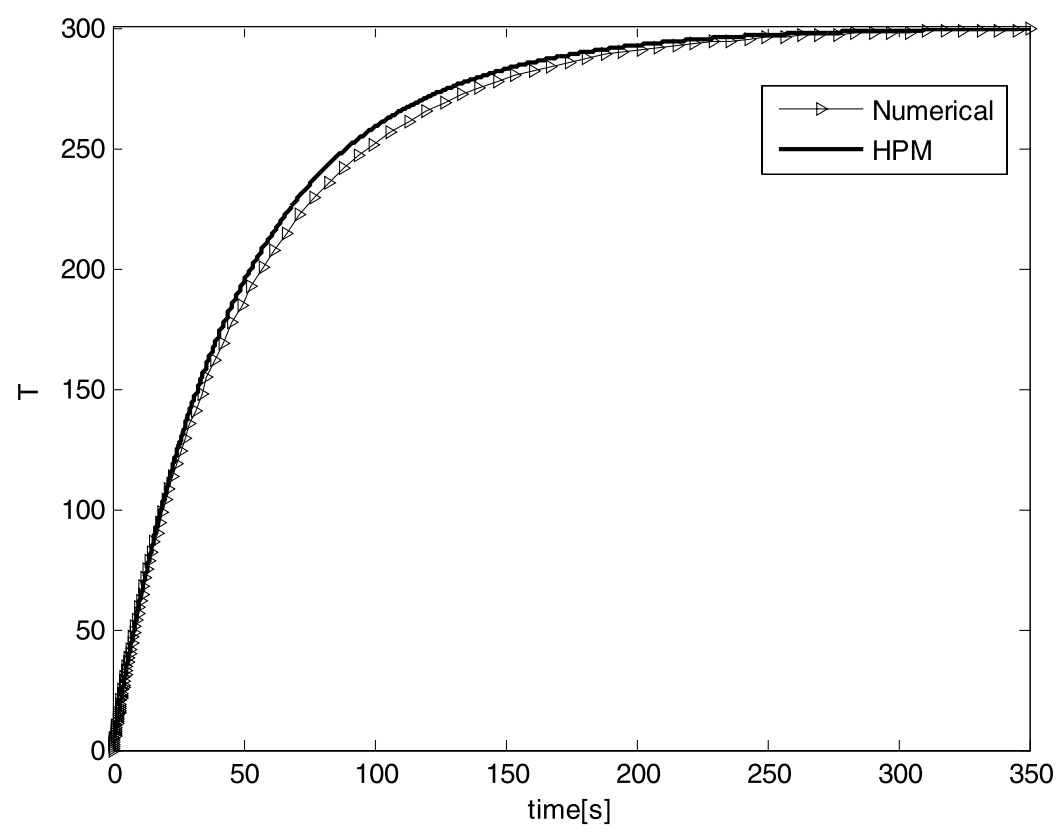

Fig. 2 Step response of an HPM based solution and the numerical one

$$
\begin{aligned}
T_{3 r d}= & T_{i}+T_{z i 3 r d} \\
= & 300-300 e^{-\frac{2}{125} t}+\left(\frac{7}{16} e^{\frac{2}{125} t}-\frac{3}{250} t+\frac{1865}{16}-117 e^{-\frac{2}{125} t}\right) e^{-\frac{2}{125} t} \\
& +3072 e^{-\frac{111}{5000} t}-1872 e^{-\frac{111}{2500} t} \\
& +\left(\frac{-1898208}{625} e^{-\frac{333}{5000} t}+\frac{4270968}{625} e^{-\frac{111}{5000} t}\right. \\
& \left.-\frac{2847312}{625} e^{-\frac{111}{5000} t}+\frac{474552}{625}\right) e^{-\frac{111}{5000} t} .
\end{aligned}
$$

The complete solution is plotted in Fig. 3. The performance of the proposed method can be seen during the transient and also in the steady state. Meanwhile the 3rd order approximation provides better result with respect to the 2 nd order. It is of hoped to achieve to a better linearized dynamic by considering the higher order terms.

\section{Deriving the equivalent transfer function of HPM based model}

So far the step response of HPM solution is found satisfactory with respect to the actual behavior. This means HPM provides an alternative good fit model for the nonlinear dynamic of course in time domain. The objective is to show how HPM produces a linearization procedure for nonlinear systems. On the other hand an HPM 


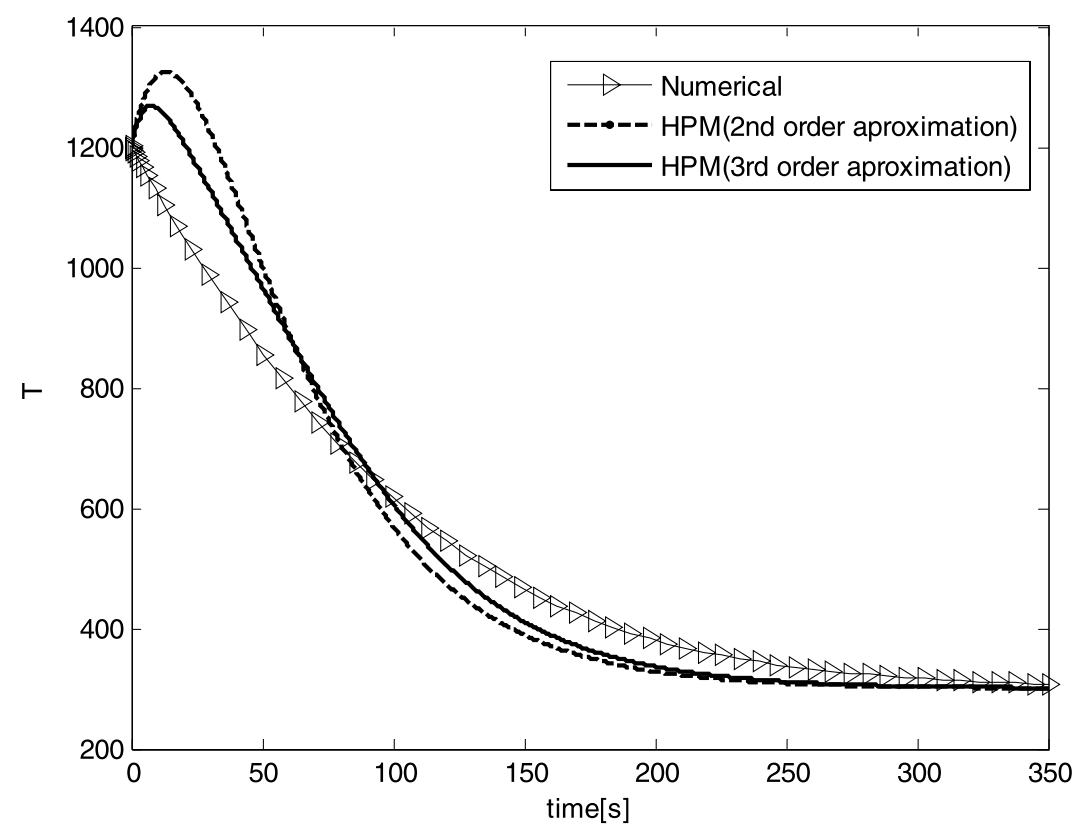

Fig. 3 Plot of complete response considering 2nd and 3rd order linearization with respect to numerical one

Fig. 4 The simplified procedure of deriving HPM linearized model

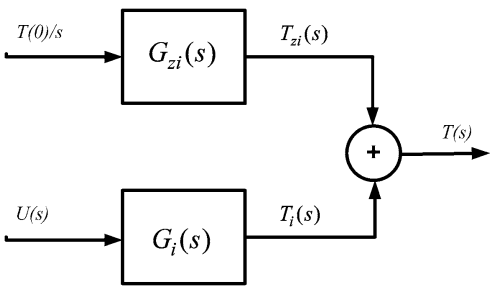

based linear model will be derived from the solution. This will initially be achieved by calculating the equivalent transfer function for the linearized thermal solution, independent from the excitation. The procedure is to take the Laplace transform of the time response analytically. It will be proceed by dividing the resultant over the input dynamic in Laplace format. This takes the effect of input out of the Laplace model. As the solution consists of two parts of:

(i) Zero input response, $T_{i}(s)$ and

(ii) Zero state response $T_{z i}$.

The model is also considered under these two circumstances. The procedure is graphically shown in Fig. 4.

The procedure consists of two parts of zero state $G_{i}(s)$, and zero input transfer $G_{z i}(s)$, functions equivalents of the heat transfer dynamic in (8). Accordingly their 
relevant responses are shown by $T_{i}(s)$ and $T_{z i}$ respectively here as:

$$
\begin{aligned}
T_{i}(s) & =\frac{6.667\left(s^{2}+0.039 s+3.69 \times 10^{-4}\right)}{s(s+0.016)^{2}(s+0.032)}, \\
T_{z i 2 n d}(s) & =\frac{1200(s+0.079)}{(s+0.0222)(s+0.0444)}, \\
T_{z i 3 r d}(s) & =\frac{1200\left(s^{3}+0.234 s^{2}+0.0163 s+4.167 \times 10^{-4}\right)}{(s+0.0222)(s+0.0444)(s+0.0666)(s+0.0888)},
\end{aligned}
$$

where $T_{z i 2 n d}(s)$ and $T_{z i 3 n d}(s)$ are the Laplace transform of approximated 2nd and 3rd order HPM based zero input responses respectively. The HPM based model (27) will be derived by dividing it to the input (step) Laplace transform. This takes the roll of input from the input Laplace transforms.

$$
\begin{gathered}
G_{i}(s)=\frac{T_{i}(s)}{T_{a}(s)}=\frac{\left[\frac{6.667\left(s^{2}+0.039 s+3.69 \times 10^{-4}\right)}{s(s+0.016)^{2}(s+0.032)}\right]}{\left[\frac{300}{s}\right]} \\
=\frac{6.667\left(s^{2}+0.039 s+3.69 \times 10^{-4}\right)}{300(s+0.016)^{2}(s+0.032)}, \\
G_{z i 2 n d}=\frac{T_{z i 2 n d}(s)}{T(0) / s}=\frac{s(s+0.079)}{(s+0.0222)(s+0.0444)}, \\
G_{z i 3 r d}=\frac{T_{z i 3 r d}(s)}{T(0) / s}=\frac{s\left(s^{3}+0.234 s^{2}+0.0163 s+4.167 \times 10^{-4}\right)}{(s+0.0222)(s+0.0444)(s+0.0666)(s+0.0888)} .
\end{gathered}
$$

\section{Simulation results}

At this section the performance of the linearized thermal model will be investigated. More precisely different kinds of input are applied to the derived model. The outcome will be compared with the numerical solution. The relevant error in presence of variety inputs is shown in Table 1.

The approximation is found satisfactory whilst small amount of error confirms the accuracy. To show the significance of the provided model it is also excited by a pseudo random sequence according to Fig. 5a. The mean and variance of the random input are $300 \mathrm{~K}$ and $100 \mathrm{~K}$, respectively. The appropriate response is shown in Fig. 5 b.

Table 1 The approximation error of HPM based linearized model

\begin{tabular}{ll}
\hline$T_{a}(\mathrm{~K})$ & Error \\
\hline 200 & 0.016 \\
250 & 0.0065 \\
300 & 0.0152 \\
350 & 0.0275 \\
400 & 0.0431 \\
\hline
\end{tabular}




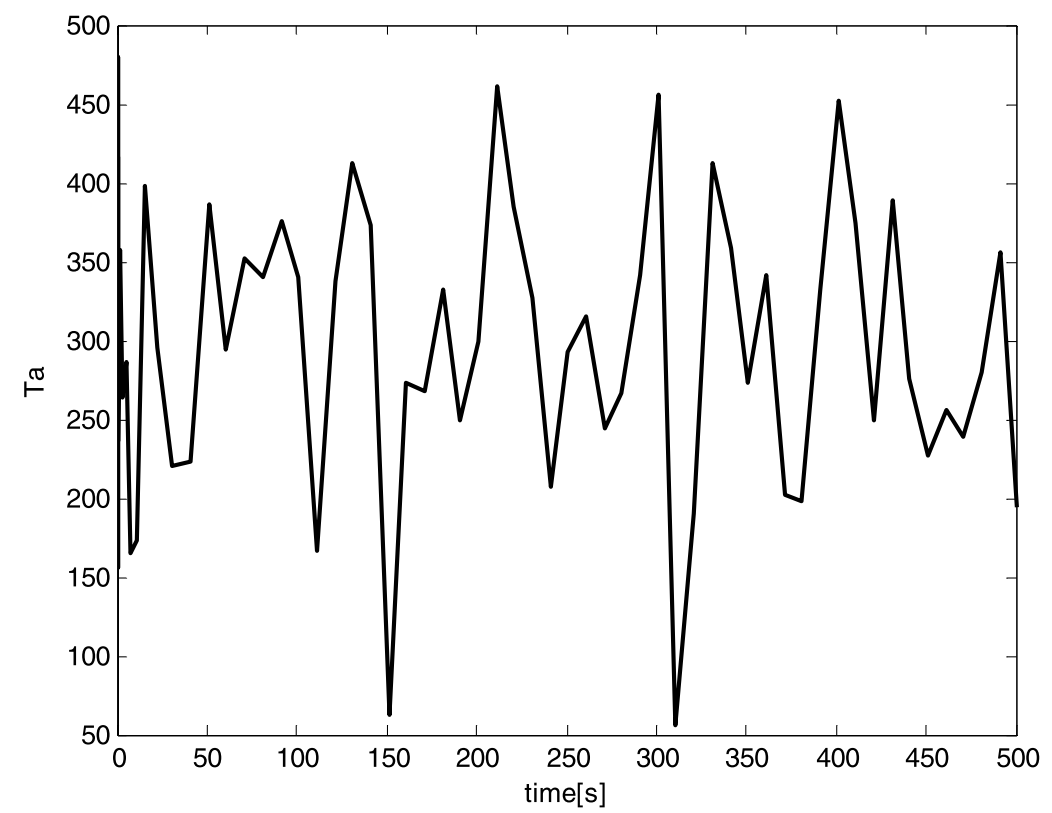

Fig. 5a A pseudo random sequence imposed to the model with mean and variance equal to $300 \mathrm{~K}$ and $100 \mathrm{~K}$ respectively

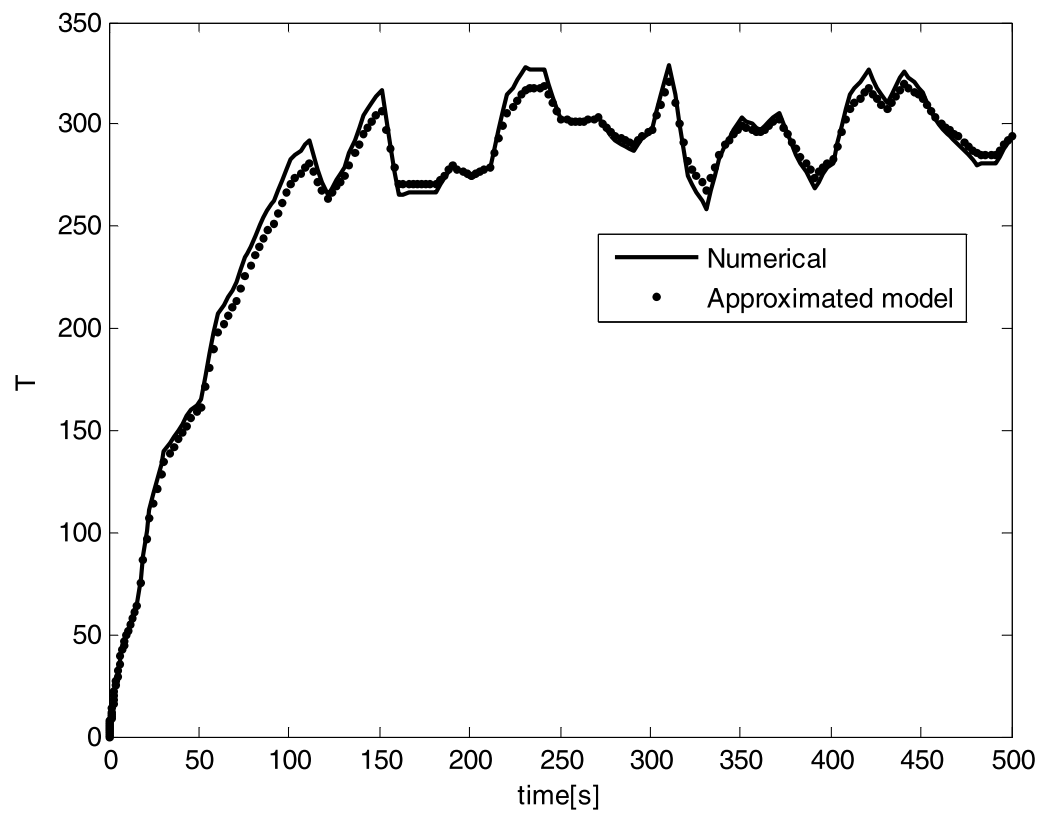

Fig. 5b The response of the model to the pseudo random input 
Furthermore the validity and quality of the proposed method will also be verified by some other process cases. This will be achieved when the provided model is used in a simple closed loop control system.

\section{A classical closed loop control}

To verify the accuracy of the linearized model it is gone under another classical test. It is assumed inside a temperature control loop with a negative unity feedback gain as shown in Fig. 6a. The result will instantaneously be compared with the actual response of nonlinear dynamic. A unit step input as an independent input together with an external excitation of initial condition is applied to those systems. The response

Fig. 6a The approximated model inside the loop
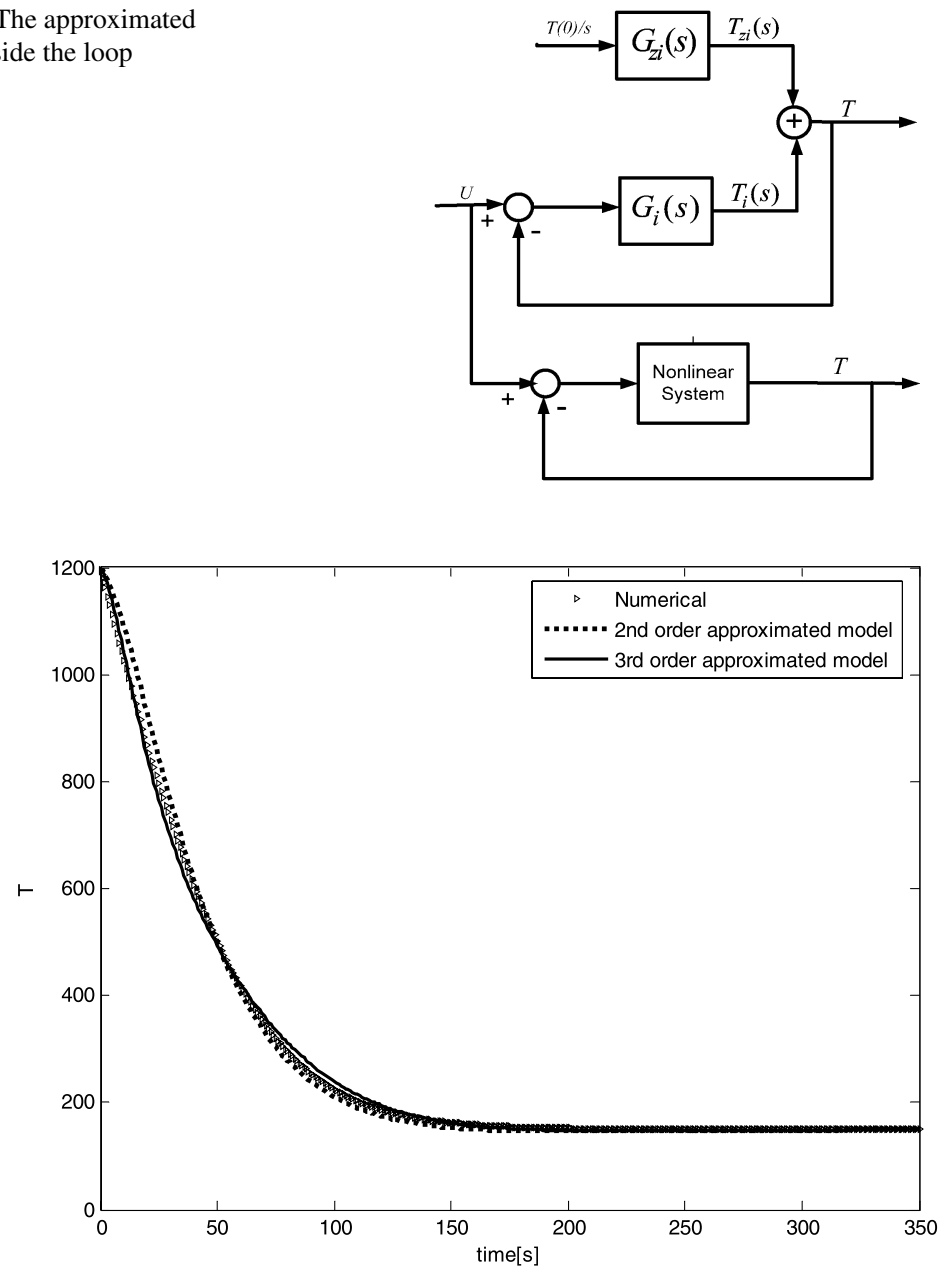

Fig. 6b The response of HPM linearized model 
Fig. 7 A control system based on the HPM linearized model
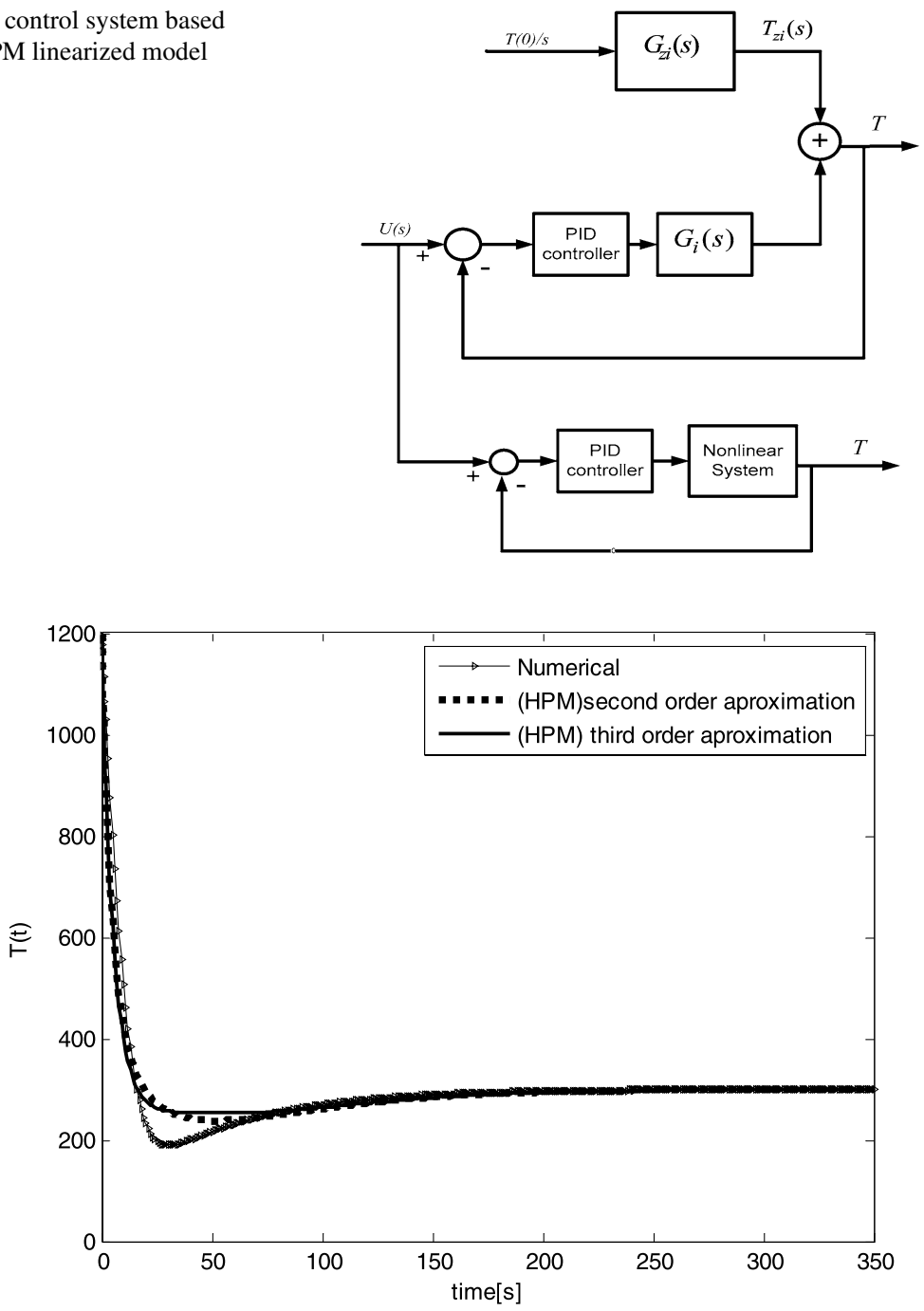

Fig. 8 Comparison between the approximated model response and that of the actual

Table 2 The error between the linearized model and the actual one

\begin{tabular}{ll}
\hline HPM linearized model & Error in percent \\
\hline 2nd order approximation & 0.5426 \\
3rd order approximation & 0.6381 \\
\hline
\end{tabular}

of 2 nd and 3rd order approximated model is also derived and compared with the numerical one in Fig. 6 b.

In essence, the 2nd and 3rd order model behaves similar. The error is $0.16 \%$ and $0.057 \%$ respectively which is almost negligible. This certifies the quality and validity 
of the proposed HPM based linearization technique. The significance of the model will be more verified whilst it is used in a PID control loop according to Fig. 7. The system is only excited by the same initial condition. The response in presence of PID controller (with the Coefficients in Table 3 in the Appendix) is shown in Fig. 8 together with the actual numerical one. Meanwhile, the error between 2nd and 3rd order linearized model is also shown in Table 2.

\section{Conclusion}

In this paper a new linearization of nonlinear dynamic is proposed. This technique is based on Homotopy Perturbation Method and has been examined on the heat transfer nonlinear differential equation. The model was interpreted in such simple terms of 2nd and 3rd order approximation. The quality of the proposed technique is shown when it is used in a classic control system. The significance of the method is also distinguished when a PID controller adjusts the transient behavior of both the actual and estimated models.

Open Access This article is distributed under the terms of the Creative Commons Attribution Noncommercial License which permits any noncommercial use, distribution, and reproduction in any medium, provided the original author(s) and source are credited.

\section{Appendix}

Table 3 PID controller coefficients

\begin{tabular}{lll}
\hline Proportional gain, $K_{p}$ & Integrator gain, $K_{i}$ & Derivative gain, $K_{d}$ \\
\hline 10 & 0.2 & 0.5 \\
\hline
\end{tabular}

Table 4 The specification of the ball

\begin{tabular}{|c|c|c|c|}
\hline The abbreviation & Showing & Unit & Value \\
\hline$m$ & Mass of the ball & $\mathrm{kg}$ & 0.26138 \\
\hline$C$ & Specific heat of the ball & $\mathrm{J} /(\mathrm{kg} \mathrm{K})$ & \\
\hline$C_{a}$ & $\begin{array}{l}\text { Specific heat of the ball (At } \\
\text { the room temperature) }\end{array}$ & $\mathrm{J} /(\mathrm{kg} \mathrm{K})$ & 420 \\
\hline$A$ & Surface area of the ball & $\mathrm{m}^{2}$ & $5.02654 \times 10^{-3}$ \\
\hline$\varepsilon$ & $\begin{array}{l}\text { Emission (Max., the small } \\
\text { parameter) }\end{array}$ & & 0.85 \\
\hline$\rho$ & Density of the ball & & 7800 \\
\hline$h$ & $\begin{array}{l}\text { The convection cooling } \\
\text { coefficient }\end{array}$ & $\mathrm{W} /\left(\mathrm{s} \mathrm{m}^{2} \mathrm{~K}\right)$ & 350 \\
\hline$\beta$ & Constant coefficient & & $9.44 \times 10^{-4}$ \\
\hline
\end{tabular}




\section{References}

1. Fakhari, A., Ganji, D., Ebrahimpour: Approximate explicit solutions of nonlinear BBMB equations by homotopy analysis method and comparison with the exact solution. Phys. Lett. A 368(1-2), 64-68 (2007)

2. Ganji, D.D., Afrouzi, G.A., Talarposhti, R.A.: Application of He's variational iteration method for solving the reaction-diffusion equation with ecological parameters. Comput. Math. Appl. 54(7-8), 1010-1017 (2007)

3. Ganji, D.D., Tari, H., Bakhshi Jooybari, M.: Variational iteration method and homotopy perturbation method for nonlinear evolution equations. Comput. Math. Appl. 54(7-8), 1018-1027 (2007)

4. Sadighi, A., Ganji, D.D.: Exact solutions of Laplace equation by homotopy-perturbation and Adomian decomposition methods. Phys. Lett. A 367(1-2), 83-87 (2007)

5. Ganji, D.D., Sadighi, A.: Application of homotopy-perturbation and variational iteration methods to nonlinear heat transfer and porous media equations. J. Comput. Appl. Math. 207(1), 24-34 (2007)

6. Liao, S.J.: An approximate solution technique not depending on small parameters: a special example. Int. J. Non-Linear Mech. 30(3), 371-380 (1995)

7. Tari, H., Ganji, D.D., Rostamian, M.: Approximate solution of K(2,2), KdV and modified KdV equations by variational iteration method, homotopy perturbation method and homotopy analysis method. Int. J. Nonlinear Sci. Numer. Simul. 8(2), 203-210 (2007)

8. Ganji, D.D.: The application of He's homotopy perturbation method to nonlinear equations arising in heat transfer. Phys. Lett. A 355(4-5), 337-341 (2006)

9. Momani, S., Suat Ertürk, V.: Solutions of non-linear oscillators by the modified differential transform method. Comput. Math. Appl. 55(4), 833 (2008)

10. Hosein Nia, S.H., Ranjbar, A.N., Ganji, D.D., Soltani, H., Ghasemi, J.: Maintaining the stability of nonlinear differential equations by the enhancement of HPM. Phys. Lett. A 372, 2855-2861 (2008)

11. He, J.-H.: Homotopy perturbation technique. Comput. Methods Appl. Mech. Eng. 178(3-4), 257-262 (1999)

12. He, J.-H.: A coupling method of a homotopy technique and a perturbation technique for non-linear problems. Int. J. Non-Linear Mech. 35, 37-43 (2000)

13. Ranjbar N., A., Hosseinnia, S.H., Soltani, H., Ghasemi, J.: A solution of Riccati nonlinear differential equation using enhanced homotopy perturbation method (EHPM). Int. J. Eng. 21(1), 27-38 (2008)

14. Chowdhury, M.S.H., Hashim, I.: Solutions of a class of singular second-order IVPs by homotopyperturbation method. Phys. Lett. A 365, 439-447 (2007)

15. Ganji, D.D., Hosseini, M.J., Shayegh, J.: Some nonlinear heat transfer equations solved by three approximate methods. Int. Commun. Heat Mass Transf. 34(8), 1003-1016 (2007)

16. Ganji, D.D., Sadighi, A.: Application of homotopy-perturbation and variational iteration methods to nonlinear heat transfer and porous media equations. Phys. Lett. A 207(1), 24-34 (2007)

17. Tari, H., Ganji, D.D., Babazadeh, H.: The application of He's variational iteration method to nonlinear equations arising in heat transfer. Phys. Lett. A 363(3), 213-217 (2007)

18. Khalil, H.: Non-linear Systems. Prentice Hall, New York (1996)

19. Vidyasagar, M.: Nonlinear Systems Analysis, 2nd edn. Prentice-Hall International, Englewood Cliffs (1993)

20. He, J.H.: An approximate solution technique depending on an artificial parameter: A special example. Commun. Non-linear Sci. Numer. Simul. 3(2), 92 (1998) 